\title{
Machine learning-XGBoost analysis of language networks to classify patients with epilepsy
}

\author{
L. Torlay $\cdot$ M. Perrone-Bertolotti $\cdot$ E. Thomas $\cdot$ \\ M. Baciu
}

Received: 21 February 2017/ Accepted: 13 April 2017/Published online: 22 April 2017

(C) The Author(s) 2017. This article is an open access publication

\begin{abstract}
Our goal was to apply a statistical approach to allow the identification of atypical language patterns and to differentiate patients with epilepsy from healthy subjects, based on their cerebral activity, as assessed by functional MRI (fMRI). Patients with focal epilepsy show reorganization or plasticity of brain networks involved in cognitive functions, inducing 'atypical' (compared to 'typical' in healthy people) brain profiles. Moreover, some of these patients suffer from drug-resistant epilepsy, and they undergo surgery to stop seizures. The neurosurgeon should only remove the zone generating seizures and must preserve cognitive functions to avoid deficits. To preserve functions, one should know how they are represented in the patient's brain, which is in general different from that of healthy subjects. For this purpose, in the pre-surgical stage, robust and efficient methods are required to identify atypical from typical representations. Given the frequent location of regions generating seizures in the vicinity of language networks, one important function to be considered is language. The risk of language impairment after
\end{abstract}

Electronic supplementary material The online version of this article (doi:10.1007/s40708-017-0065-7) contains supplementary material, which is available to authorized users.

L. Torlay $\cdot$ M. Perrone-Bertolotti $\cdot$ M. Baciu

CNRS LPNC UMR 5105, Univ. Grenoble Alpes,

380000 Grenoble, France

E. Thomas

Laboratoire INSERM U1093, Université de Bourgogne,

21000 Dijon, France

M. Baciu $(\square)$

LPNC, UMR CNRS 5105, BSHM, Université Pierre

Mendès-France, BP 47, 38040 Grenoble Cedex 09, France

e-mail: monica.baciu@univ-grenoble-alpes.fr surgery is determined pre-surgically by mapping language networks. In clinical settings, cognitive mapping is classically performed with fMRI. The fMRI analyses allowing the identification of atypical patterns of language networks in patients are not sufficiently robust and require additional statistic approaches. In this study, we report the use of a statistical nonlinear machine learning classification, the Extreme Gradient Boosting (XGBoost) algorithm, to identify atypical patterns and classify 55 participants as healthy subjects or patients with epilepsy. XGBoost analyses were based on neurophysiological features in five language regions (three frontal and two temporal) in both hemispheres and activated with fMRI for a phonological (PHONO) and a semantic (SEM) language task. These features were combined into 135 cognitively plausible subsets and further submitted to selection and binary classification. Classification performance was scored with the Area Under the receiver operating characteristic curve (AUC). Our results showed that the subset SEM_LH BA_47-21 (left fronto-temporal activation induced by the SEM task) provided the best discrimination between the two groups (AUC of $91 \pm 5 \%$ ). The results are discussed in the framework of the current debates of language reorganization in focal epilepsy.

Keywords Language - Epilepsy - Atypical - Machine learning $\cdot$ ML $\cdot$ Extreme Gradient Boosting $\cdot$ XGBoost

\section{Introduction}

Focal epilepsy is characterized by symptoms induced by lesion or dysfunction of a specific cerebral region, the 'epileptic zone' (EZ) [1]. Given the location of the EZ 
within or in the vicinity of language networks, patients are more or less impaired for language abilities but they clearly show reorganization of language networks based on functional plasticity $[2,3]$. Compared to typical left hemisphere representation of language observed in the majority of healthy subjects [4], patients with epilepsy show a higher frequency of atypical language representation, with both inter- and intra-hemispheric reorganization (see [3] for a review). In addition, an important percentage of patients with epilepsy become resistant to anti-epileptic medication and surgery is undertaken to remove the EZ and stop seizures $[5,6]$. In the pre-surgical phase, language mapping is required to identify language regions that must be preserved during surgery to avoid cognitive deficit. Mapping provides information in terms of language specialization, either at a hemispheric or at a regional level, as both levels provide specific patterns of reorganization. The global hemispheric specialization for language (and other cognitive functions) is clinically assessed with the Wada test $[7,8]$, while language networks are generally defined at an inter- and intra-hemispheric level with noninvasive methods such as functional MRI. Functional MRI maps language in patients with epilepsy by using a large variety of tasks and protocols [9-17] even if there is no clear consensus on the most appropriate task or panel of tasks to be used. In clinical practice, phonological and lexico-semantic tasks are generally used to maximize the amount of relevant information for language network activity. The identification of language patterns, mostly atypical in patients, requires precision (in terms of language tasks and analysis of data) to correctly describe the reorganized networks and to avoid deficits after surgery.

In terms of data analysis, we generally determine (qualitatively/inspection or quantitatively/statistically) for a given task and a given patient, the language network and its functional hemispheric or regional lateralization [17]. This individual analysis has inherent limits linked to the high intra- and inter-patient variability of activation, giving rise to poor statistical power and subjective interpretation $[18,19]$. These limits are particularly important in the case of patients who need surgery and show a high risk of postsurgical sequels if the region responsible for seizures is located in the vicinity of reorganized language networks, which are not correctly identified before surgery. In sum, there is a strong need to develop robust statistical and objective approaches to identify language networks in patients with epilepsy. Functional MRI is a very helpful tool to highlight activated regions but the information on this activation is not sufficient [20]. Specifically, this activation needs to be further processed and included into more robust statistical analyses, to obtain robust results allowing for a clearer distinction between patients with reorganized cognitive networks and healthy subjects with typical representations of cognitive functions. Due to the lack of statistical power when trying to validate differences between single-patient fMRI measures and a group of heathy subjects, robust statistical methods should be involved.

The main objective of this study is to evaluate an objective method to distinguish patients and healthy people, based on language networks mapped with fMRI, and by using a machine learning (ML) approach. Previous results from a range of cognitive studies [21-24] showed successful use of ML classification. In patients with epilepsy, an ML approach based on a probabilistic regression method was used on fMRI data to evaluate the hemispheric specialization for language before surgery [18]. The authors showed successful classification (96\%) with dissociation between typical (i.e., left hemisphere predominance) and atypical patterns of lateralization. Moreover, patients with atypical patterns (i.e., right hemisphere dominant or bilateral representation) were successfully identified $(82 \%)$. An important advantage of the ML approach is that predetermined parameters (a priori threshold value settings as classically used in fMRI analysis) are not necessary, removing the subjective dimension of analyses and interpretations. Other authors have used ML on data concerning the integrity of white matter fibers to predict the surgical outcome in patients with epilepsy [25]. This approach was able to distinguish patients with epilepsy from normal controls with $80 \%$ accuracy, and predict the surgical outcome for patients, with $70 \%$ accuracy. ML classification requires input features or dimensions. In fMRI, these features are represented by the amount of the blood oxygen level-dependent (BOLD) signals in regions of interest or by lateralization indices, as used by [18]. The latter are calculated with BOLD signal values measured in homologues (right and left hemisphere) regions of interest. fMRI activity and BOLD signals strongly depend on psycholinguistic features such as language operation (phonology and semantic) and tasks used during fMRI assessment. Ideally, a fine-grained representation of language networks in patients implies the use of a panel of language tasks. This is difficult to apply in clinical practice due to practical reasons (short duration of the fMRI protocol, tasks should be easy to perform by patients). Consequently, a compromise has to be found between the amount of information in terms of language networks and pragmatic criteria inherent to work with patients. In this framework, it is accepted that the essential information concerning language networks in patients with epilepsy is obtained by using a phonological and semantic task (see, for instance, [3] and [15]).

In this current study, we applied a ML classifier, the Extreme Gradient Boosting algorithm (XGBoost) [26] in order to discriminate the fMRI from epileptic patients and 
healthy subjects. This particular method was chosen due to its significant advantages: (a) dealing with missing values, (b) requiring data scaling, (c) implying a computationally efficient variant of gradient boosting algorithm [27], (d) providing satisfactory results in ML competitions [28] and was successfully used in other studies and domains (see [29, 30]). Using XGBoost, we expected to identify robust patterns of language representation which are able to distinguish patients and healthy people. To our knowledge, there are no studies using XGBoost to objectively classify two populations based on their neurophysiological features. Specifically, we examined 55 participants who underwent fMRI and performed two language tasks-a semantic and phonological one, which activate classical language regions [15, 16, 31-33]. Based on the activation, we defined 20 features, as follows: five fronto-temporal (FT) regions (BA, Brodmann Area; BA21, BA 22, BA 44, BA 45 and BA 47), delineated in each (left, $\mathrm{LH}$; right, $\mathrm{RH}$ ) or both (bilateral; LH-RH) hemispheres, and for each language task (SEM, PHONO). We expect that differences between healthy subjects and patients with epilepsy would reveal atypical patterns of language representation in the damaged brains of patients. The atypical patterns might also reflect 'sensitive-to-surgery' regions that must be preserved during surgery to avoid language deficits.

\section{Material and methods}

\subsection{Participants}

We examined 55 participants: 16 patients with focal epilepsy and 39 healthy controls. Patients showed various anatomical locations of the EZ, and as indicated in Table 1, they were right- and left-handed. All were native French speakers and had normal or corrected-to-normal vision. Healthy volunteers had no history of neurological or psychiatric disorders. Participants gave informed written consent, and the study was approved by the local ethics committee (CPP no 09-CHUG-14, 04/06/2009).

Table 1 Demographic information of participants, patients (TLE, patient with epilepsy with left temporal lobe epilepsy) and healthy volunteers (controls)

\begin{tabular}{lllll}
\hline & $N$ & Age mean $(\mathrm{SD})$ & Gender & Handedness \\
\hline TLE & 16 & $35.3 \pm 11.1$ & $9 \mathrm{M}-7 \mathrm{~F}$ & $1 \mathrm{~L}-15 \mathrm{R}$ \\
Controls & 39 & $26.5 \pm 3.7$ & $18 \mathrm{M}-21 \mathrm{~F}$ & $15 \mathrm{~L}-24 \mathrm{R}$ \\
\hline
\end{tabular}

For each group, we mentioned the number of participants $(N)$, the mean age and standard deviation $(S D)$, the gender $(F$, female; $M$, male) and the handedness (right-handed, $R$; left-handed, $L$ )

\subsection{Stimuli and tasks}

The experimental protocol used during fMRI examination is described in detail in [15]. Two language tasks were used in two separate runs, a phonological (PHONO) and a semantic (SEM) task, each one also including a control visual condition (without language demands). Each task comprised 'language' and 'control' conditions. The PHONO language condition was performed with pseudowords. Participants were instructed to detect a target phoneme (phoneme detection task). The SEM language condition of SEM run was performed using words with participants being instructed to judge whether items designated living or non-living entities (categorization task). The control condition was identical for the two runs and was performed using unreadable words (font Karalyn Patterson) with participants being instructed to judge the height of characters (visual detection task). Stimuli generated by the E-Prime software (E-prime Psychology Software Tools Inc., Pittsburgh, USA) were written in white 'Courier New' font size 40, centered on the middle of a black screen and lasted $2.5 \mathrm{~s}$ each.

\subsection{Functional MRI paradigm}

A pseudo-randomized event-related fMRI paradigm was optimized [34] for 60 events, and 35 additional null events were used for each run (PHONO and SEM runs). The null events were added in order to provide an appropriate baseline measure [1] and consisted of a white fixation cross-displayed in the center of the black screen. The interstimulus interval was $2.5 \mathrm{~s}$. The run duration was $8 \mathrm{~min}$ $40 \mathrm{~s}$.

\subsection{MR acquisition}

The experiment was performed in a whole-body 3T MR scanner (Bruker MedSpec S300) with $40 \mathrm{mT} / \mathrm{m}$ gradient strength at MR facility. For functional runs, the manufacturer-provided gradient-echo/T2*-weighted EPI method was used. Thirty-nine adjacent axial slices parallel to the bi-commissural plane were acquired in an interleaved mode. Slice thickness was $3.5 \mathrm{~mm}$. During each run, the cerebral volume was measured 150 times. The in-plane voxel size was $3 \times 3 \mathrm{~mm}(216 \times 216 \mathrm{~mm}$ field of view acquired with a $72 \times 72$ pixel data matrix, reconstructed with zero filling to $128 \times 128$ pixels). The main sequence parameters were: $\mathrm{TR}=2.5 \mathrm{~s}, \quad \mathrm{TE}=40 \mathrm{~ms}$, flip angle $=77^{\circ}$. To correct images for geometric distortions induced by local B0 inhomogeneity, a B0 field map was obtained from two gradient-echo datasets acquired with a standard 3D FLASH sequence $(\Delta \mathrm{TE}=9.1 \mathrm{~ms})$. The field map was used during data processing. A T1-weighted high- 
resolution three-dimensional anatomical volume was also acquired, by using a 3D-modified driven equilibrium Fourier transform (MDEFT) sequence (field of view: $256 \times 224 \times 176 \mathrm{~mm}$; resolution: $1.333 \times 1.750 \times$ $1.375 \mathrm{~mm}$; acquisition matrix: $192 \times 128 \times 128$ pixels; reconstruction matrix: $256 \times 128 \times 128$ pixels).

\subsection{Spatial preprocessing of fMRI data}

Data analysis was performed by using the general linear model, GLM [35] for event-related designs with SPM12 (Wellcome Department of Imaging Neuroscience, London, UK, www.fil.ion.ucl.ac.uk/spm) implemented in MATLAB (MathWorks Inc., Natick, MA, USA). Images were spatially preprocessed. First, the functional volumes were time-corrected with the 19th slice as reference (the acquired brain volume was composed of 39 slices) to correct artifacts caused by the delay of time acquisition between slices. Subsequently, all volumes were realigned to correct for head motion, by using a rigid body transformation. T1-weighted anatomical volume was co-registered to mean images created by the realignment procedure and was normalized within the MNI space. Anatomical normalization parameters were used for the normalization of functional volumes. Each functional volume was smoothed by a Gaussian kernel of $8 \mathrm{~mm}$ FWHM (Full Width at Half Maximum). Finally, time series for each voxel were high-pass filtered $(1 / 128 \mathrm{~Hz}$ cutoff) to remove low-frequency noise and signal drift.

\subsection{Statistical analyses of fMRI data}

Statistical analyses were subsequently performed on the preprocessed data. For each participant, each task (PHONO and SEM) was declared as a specific fMRI run. Thus, for each run PHONO or SEM, we included two regressors, PHONO (task) and Control-PHONO, and SEM (task) and Control-SEM, respectively. Each of them was convolved with a canonical hemodynamic response function (HRF). Movement parameters derived from the realignment corrections (three translations and three rotations) were included into the design matrix as additional factors of no interest. The GLM was then used to generate the parameter estimates of activity for each voxel, each condition and each participant. Statistical parametric maps were generated from the linear contrasts between the HRF parameter estimates for the four experimental conditions (i.e., task and control for each run). The spatial resolution of statistical parametric maps was the same as the spatial resolution of functional MR images $(3 \times 3 \times 3.5 \mathrm{~mm})$. The statistical analysis was performed at a first level (Individual level) by calculating the main contrasts that were PHONO (task) versus Control-PHONO and SEM (task) versus ControlSEM. These contrasts allowed us to identify language networks for phonology and semantic processes.

\subsection{ROI construction and extraction of the \% MR signal (BOLD)}

In accordance with our previously reported results from group analyses and based on previous literature on PHONO and SEM processing [2-4], we determined ten symmetrical frontal and temporal regions of interest (ROI), five in the left $(\mathrm{LH})$ and five in the right hemisphere (RH). ROIs were defined based on the WFU PickAtlas toolbox (https://www. nitrc.org/projects/wfu_pickatlas/) from the Brodmann Area (BA) labeling. ROIs taken into account were, bilaterally, the inferior frontal gyrus pars opercularis, BA 44, pars triangularis, BA 45 and pars orbitalis, BA 47; middle temporal, BA 21 and superior temporal BA 22 gyri. For each ROI, each participant and each task (PHONO and SEM), the \% of MR signal intensity variation (average of all voxels within a specific ROI) was measured. We defined 20 features for use in the ML classification approach (cf. 2.8 - the $\%$ of BOLD variation within the considered ROIs for PHONO (five ROI in the LH and five ROI in the $\mathrm{RH}$ ) and for SEM (five ROI in the LH and five ROI in the $\mathrm{RH})$.

\subsection{Machine learning}

The ML approach aims to find a relationship between an input $X=\left\{x_{1}, x_{2}, \ldots, x_{N}\right\}$ and an output $Y$. In our case, we inferred the relationship between the fMRI BOLD signal values and the participant condition (healthy; patient with epilepsy). In other words, we determined whether a participant is a patient with epilepsy or a healthy subject based on fMRI activation. More precisely, we aimed at determining the best combination(s) of features (according to region, hemisphere and task) showing the most predictive power in this binary classification. We used the XGBoost algorithm, an implementation of the gradient-boosted decision trees (GBDT) for this purpose. Assembly algorithms create and combine a high number of individually weak but complementary classifiers, to produce a robust estimator. This combination could be made in two ways: bagging (random forests) and boosting. The gradient boosting is built sequentially. Indeed, a new weak learner is constructed to be maximally correlated with the negative gradient of the loss function associated with the whole assembly for each iteration [36]. XGBoost belongs to the group of widely used tree learning algorithms [37]. A decision tree allows making prediction on an output variable based on a series of rules arranged in a tree-like structure. They consist of a series of split points, the nodes, 
in terms of the value of an input feature. The last node is a leaf and gives us the specific value of the output variable. Tree learning algorithms do not require linear features or linear interactions between features. They are significantly better classifiers than other algorithms (see [38]). Moreover, XGBoost, a type of gradient boosting, has two major improvements: (a) speeding up the tree construction and (b) proposing a new distributed algorithm for tree searching. All participants (samples) were described by the set of 20 features mentioned above. We combined these features into specific cognitively plausible subsets in order to reduce the number of combinations in the feature selection step (cf. 2.8.1). The entire procedure used in this study is presented in detail in Supplementary Material. We had eight missing among 1100 values $(0.7 \%)$ from eight healthy participants for two features, BA 44 RH_SEM and the BA 44 RH_PHONO. We did not perform imputation or scaling on the data.

\subsubsection{Feature selection method}

The goal of feature selection was to choose a subset $X_{S}$ of $X$ that can predict $Y$ with the best performance at minimal computational cost. Another objective was to gain insight into the underlying processes which generated the data.

There are three main categories of feature selection algorithms: filter, wrapper and embedded. In the present study, we have focused on the filter and wrapper methods. Filter methods are computed fast and provide a feature ranking in order to remove irrelevant features. Although some of them are multivariate, such as correlation-based feature selection (CFS), they do not involve a learning algorithm and can miss useful features. Wrapper approaches use a given classification algorithm for the evaluation of a specific subset of features by training and testing it with cross-validation. The space of all feature subsets is generated by the strategy defined above. An exhaustive search with these features involves around one million combinations $\left(2^{20}\right)$. After evaluation, some filter methods (low variance, Fisher score, CFS, Laplacian score, spectral score) and the forward (SFS) and backward selection (SBS) wrapper methods were considered unsuccessful (i.e., with no feature subset stability and with no significance of the performance metrics). Filter methods were tested with scikit-feature Python libraries implementation [39]. SFS and SBS wrapper methods were performed with Mlxtend Python libraries [40].

Thus, we decided to perform another wrapper method, a reduced exhaustive search among a selection of 135 feature subsets. We did not perform an exhaustive search with the $2^{20}$ combinations of features, which would have been too long to compute and would have been prone to overfitting. These 135 different combinations of features were chosen and grouped in nine 'thematic sets' (see Table 2) based on ROI, hemisphere, and task. They are specified as follows: (a) three for SEM including a left hemisphere thematic set (with 15 subsets), a right hemisphere thematic set (including 15 subsets) and a bilateral thematic set (including 15 subsets); (b) three for PHONO including a left hemisphere thematic set (with 15 subsets), a right hemisphere thematic set (including 15 subsets) and a bilateral thematic set (including 15 subsets), and (c) three for SEM + PHONO including a left hemisphere thematic set (with 15 subsets), a right hemisphere thematic set (including 15 subsets) and a bilateral thematic set (including 15 subsets). These 135 feature subsets were combinations of the ROIs reflecting plausible patterns of language organization and reorganization based on literature results [3, 41, 42]. They varied according to ROIs (only frontal, only temporal or both), hemisphere (only left, only right or both hemispheres) and task (PHONO only, SEM only or PHONO + SEM).

\subsubsection{Classification method}

The Extreme Gradient Boosting (XGBoost) algorithm [43] was used for classification. It was implemented using the scikit-learn [40] Python libraries for all ML processes. Parameters for the algorithm were fixed (cf. Table 3) and not optimized by a grid search for the whole ML process (see [44]). Four parameters out of fifteen were set to particular values. The learning rate was typically set to 0.01 (default 0.3) as small values lead to much better generalization [45]. The number of boosted trees usually between some hundreds and thousands was set to 1200 (estimators). To prevent overfitting, the subsample was set to 0.7 (default 1). This added randomness and made the training robust to noise. The maximum depth of a tree was set to 3 (default 6) to reduce the model complexity.

\subsubsection{Validation strategy}

The validation strategy is commonly used to prevent overfitting and to have a good assessment of model validity [44]. As illustrated in Fig. 1, we have used a nested crossvalidation scheme with an outer Monte Carlo cross-validation (MCCV) (see [46, 47]), also called random subsampling, repeated twelve times in order to reduce variance and an inner $k$-fold cross-validation ( $k$-fold $\mathrm{CV}$ ) for feature selection with $\mathrm{k}=5$ (see [44, 45]). Feature selection must be done inside each training set of the outer cross-validation that estimates the performance of the model fitting approach [48]. First, we randomly select, without replacement, $80 \%$ of our data to form the training set. The remaining $20 \%$ was the validation set including a balanced number of epileptic and healthy subjects. Each participant 
Table 2 A total of 135 subsets were evaluated

\begin{tabular}{|c|c|c|c|c|c|c|}
\hline Task & Subset & $\begin{array}{l}\text { Fronto- } \\
\text { temporal } \\
\text { regions } \\
(\mathrm{FT})\end{array}$ & Left hemisphere & Right hemisphere & Bilateral & \\
\hline SEM only & 1 & Partial F & BA $47 \mathrm{LH}$ & BA 47RH & BA 47LH & BA 47RH \\
\hline \multirow{14}{*}{$\begin{array}{l}\text { or PHONO only } \\
\text { or } \\
\text { SEM + PHONO }\end{array}$} & 2 & Partial F & BA 44LH, BA 45LH, & BA 44RH, BA 45RH, & BA $44 \mathrm{LH}, \mathrm{BA} 45 \mathrm{LH}$, & BA 44RH, BA 45RH, \\
\hline & 3 & Total F & $\begin{array}{l}\text { BA 44LH, BA } 45 \mathrm{LH}, \\
\text { BA 47LH }\end{array}$ & $\begin{array}{l}\text { BA 44RH, BA 45RH, } \\
\text { BA 47RH }\end{array}$ & $\begin{array}{l}\text { BA } 44 \mathrm{LH}, \mathrm{BA} 45 \mathrm{LH} \text {, } \\
\text { BA } 47 \mathrm{LH}\end{array}$ & $\begin{array}{l}\text { BA 44RH, BA 45RH, } \\
\text { BA 47RH }\end{array}$ \\
\hline & 4 & Partial T & BA $21 \mathrm{LH}$ & BA $21 \mathrm{RH}$ & BA $21 \mathrm{LH}$ & BA $21 \mathrm{RH}$ \\
\hline & 5 & Partial T & BA $22 \mathrm{LH}$ & BA $22 \mathrm{RH}$ & BA $22 \mathrm{LH}$ & BA $22 \mathrm{RH}$ \\
\hline & 6 & Total T & BA $21 \mathrm{LH}, \mathrm{BA} 22 \mathrm{LH}$ & BA $21 \mathrm{RH}, \mathrm{BA} 22 \mathrm{RH}$ & BA $21 \mathrm{LH}, \mathrm{BA} 22 \mathrm{LH}$ & BA $21 \mathrm{RH}, \mathrm{BA} 22 \mathrm{RH}$ \\
\hline & 7 & Partial FT & BA $21 \mathrm{LH}, \mathrm{BA} 47 \mathrm{LH}$ & BA $21 \mathrm{RH}, \mathrm{BA} 47 \mathrm{RH}$ & BA 21LH. BA 47LH & BA 21RH. BA 47RH \\
\hline & 8 & Partial FT & BA 22LH, BA 47LH & BA $22 \mathrm{RH}, \mathrm{BA} 47 \mathrm{RH}$ & BA 22LH, BA 47LH & BA $22 \mathrm{RH}, \mathrm{BA} 47 \mathrm{RH}$ \\
\hline & 9 & Partial FT & $\begin{array}{l}\text { BA } 21 \mathrm{LH}, \mathrm{BA} 22 \mathrm{LH}, \\
\text { BA } 47 \mathrm{LH}\end{array}$ & $\begin{array}{l}\text { BA } 21 R H, B A ~ 22 R H, \\
\text { BA } 47 R H\end{array}$ & $\begin{array}{l}\text { BA } 21 \mathrm{LH}, \text { BA } 22 \mathrm{LH}, \\
\text { BA } 47 \mathrm{LH}\end{array}$ & $\begin{array}{l}\text { BA } 21 \mathrm{RH}, \mathrm{BA} 22 \mathrm{RH} \text {, } \\
\text { BA } 47 \mathrm{RH}\end{array}$ \\
\hline & 10 & Partial FT & $\begin{array}{l}\text { BA } 21 \mathrm{LH}, \mathrm{BA} 44 \mathrm{LH}, \\
\text { BA } 45 \mathrm{LH}\end{array}$ & $\begin{array}{l}\text { BA } 21 R H, B A 44 R H, \\
\text { BA 45RH }\end{array}$ & $\begin{array}{l}\text { BA } 21 \mathrm{LH}, \mathrm{BA} 44 \mathrm{LH} \text {, } \\
\text { BA } 45 \mathrm{LH}\end{array}$ & $\begin{array}{l}\text { BA } 21 \mathrm{RH}, \mathrm{BA} 44 \mathrm{RH} \text {, } \\
\text { BA } 45 \mathrm{RH}\end{array}$ \\
\hline & 11 & Partial FT & $\begin{array}{l}\text { BA } 22 \mathrm{LH}, \mathrm{BA} 44 \mathrm{LH}, \\
\text { BA } 45 \mathrm{LH}\end{array}$ & $\begin{array}{l}\text { BA } 22 R H, B A \\
\text { BA } 45 R H\end{array}$ & $\begin{array}{l}\text { BA } 22 \mathrm{LH}, \mathrm{BA} 44 \mathrm{LH}, \\
\text { BA } 45 \mathrm{LH}\end{array}$ & $\begin{array}{l}\text { BA } 22 R H, B A 44 R H, \\
\text { BA 45RH }\end{array}$ \\
\hline & 12 & Partial FT & $\begin{array}{l}\text { BA 21LH, BA 22LH, } \\
\text { BA 44LH, BA } \\
\text { 45LH }\end{array}$ & $\begin{array}{l}\text { BA 21RH, BA 22RH, } \\
\text { BA 44RH, BA } \\
\text { 45RH }\end{array}$ & $\begin{array}{l}\text { BA 21LH, BA 22LH, } \\
\text { BA 44LH, BA } \\
\text { 45LH }\end{array}$ & $\begin{array}{l}\text { BA 21RH, BA 22RH, } \\
\text { BA 44RH, BA } \\
\text { 45RH }\end{array}$ \\
\hline & 13 & Partial FT & $\begin{array}{l}\text { BA 21LH, BA 44LH, } \\
\text { BA 45LH, BA } \\
\text { 47LH }\end{array}$ & $\begin{array}{l}\text { BA 21RH, BA 44RH, } \\
\text { BA 45RH, BA } \\
\text { 47RH }\end{array}$ & $\begin{array}{l}\text { BA 21LH, BA 44LH, } \\
\text { BA 45LH, BA } \\
\text { 47LH }\end{array}$ & $\begin{array}{l}\text { BA 21RH, BA 44RH, } \\
\text { BA 45RH, BA } \\
\text { 47RH }\end{array}$ \\
\hline & 14 & Partial FT & $\begin{array}{l}\text { BA 22LH, BA 44LH, } \\
\text { BA 45LH, BA } \\
\text { 47LH }\end{array}$ & $\begin{array}{l}\text { BA 22RH, BA 44RH, } \\
\text { BA 45RH, BA } \\
\text { 47RH }\end{array}$ & $\begin{array}{l}\text { BA 22LH, BA 44LH, } \\
\text { BA 45LH, BA } \\
\text { 47LH }\end{array}$ & $\begin{array}{l}\text { BA 22RH, BA 44RH, } \\
\text { BA 45RH, BA } \\
\text { 47RH }\end{array}$ \\
\hline & 15 & Total FT & $\begin{array}{c}\text { BA 21LH, BA 22LH, } \\
\text { BA 44LH, BA } \\
\text { 45LH, BA 47LH }\end{array}$ & $\begin{array}{l}\text { BA 21RH, BA 22RH, } \\
\text { BA 44RH, BA } \\
\text { 45RH, BA 47RH }\end{array}$ & $\begin{array}{l}\text { BA 21LH, BA 22LH, } \\
\text { BA 44LH, BA } \\
\text { 45LH, BA 47LH }\end{array}$ & $\begin{array}{c}\text { BA 21RH, BA 22RH, } \\
\text { BA 44RH, BA } \\
\text { 45RH, BA 47RH }\end{array}$ \\
\hline
\end{tabular}

Fifteen subsets were based on combinations of fronto-temporal (FT) regions according to hemisphere and task and defined as follows: (a) only frontal regions (partial subsets 1-2 and total subset 3); (b) only temporal regions (partial subsets 4-5 and total subset 6), and (c) combination of frontal and temporal regions (partial subsets 7-14 and total subset 15). These subsets were evaluated for three thematic sets according to task (semantic only, SEM only; phonological only, PHONO only; semantic and phonological combined, SEM + PHONO) and hemisphere (left hemisphere, right hemisphere and bilateral—both hemispheres)

Table 3 Results obtained for the selected subset SEM (semantic) LH (left hemisphere) BA 21 and BA47 in terms of AUC as the performance metric for each iteration of the outer MCCV, using the

\begin{tabular}{lccccccccccccc}
\hline $\begin{array}{l}\text { Iteration } \\
\text { number }\end{array}$ & 1 & 2 & 3 & 4 & 5 & 6 & 7 & 8 & 9 & 10 & 11 & 12 \\
\hline Subset & SEM & SEM & SEM & SEM & SEM & SEM & SEM & SEM & SEM & SEM & SEM & SEM \\
selected & L21 & L21 & L21 & L21 & L21 & L21 & L21 & L21 & L21 & L21 & L21 & L21 \\
& L47 & L47 & L47 & L47 & L47 & L47 & L47 & L47 & L47 & L47 & L47 & L47 \\
AUC (\%) & 93.75 & 87.50 & 87.50 & 93.75 & 93.75 & 93.75 & 100 & 83.33 & 83.33 & 93.75 & 87.50 & 100 \\
\hline
\end{tabular}

appears in either the learning set or test set. Then fivefold cross-validation (CV) [49] was used only on the training set. It was split into five data blocks, four used for an inner training and the remaining one for the inner test. This was
XGBoost algorithm (n_estimators $=1200$, learning rate $=0.01$, subsample $=0.7$, max_depth $=3$ ) 
Fig. 1 Illustration of the validation schema, using outer Monte Carlo cross-validation (MCCV)

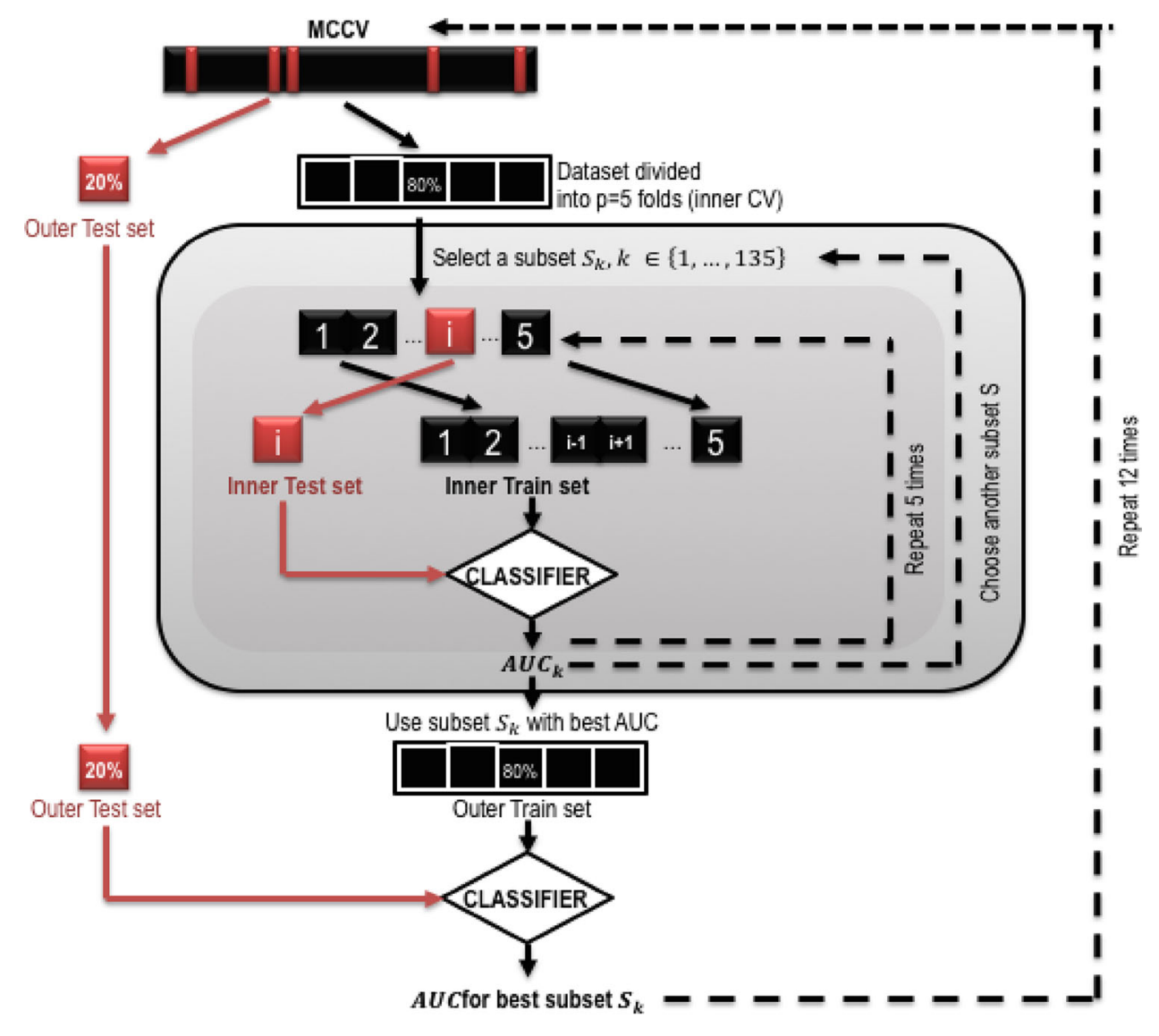

\section{Results}

on the validation set that was held out from the feature selection step. All splits were performed in a stratified way to get the same ratio of patients with epilepsy. In order to assure stability of feature selection and get correct generalization of the classification performance, we repeated this process twelve times by randomly shuffling the dataset before splitting into training and validation sets. We finally obtained twelve performance metrics (cf. Table 3) for twelve feature subsets, based on a distribution of samples.

\subsubsection{Metrics}

The predictive power of a classifier was scored by the area under the receiver operating characteristic curve (AUC) as recommended by Provost [50-52]. The AUC can be interpreted as the probability that a classifier ranks a randomly chosen positive instance higher than a randomly chosen negative one (assuming 'positive' ranks higher than 'negative'). The receiver operating curve (ROC) was the true positive rate plotted as a function of the false positive rate where the positive condition was to be a patient with epilepsy. It represents the performance of the model on a two-dimensional curve. The AUC value then reduces it to a number. A perfect model would score an AUC of $100 \%$ while a random classification would score $50 \%$.
As illustrated in Table 3 and Fig. 2, the feature subset Semantic left hemisphere BA21_BA47 was selected each time among 135 candidates, with an AUC mean of $91 \pm 5 \%$ on the validation set. The ML process showed a

\section{Distribution of classification results}

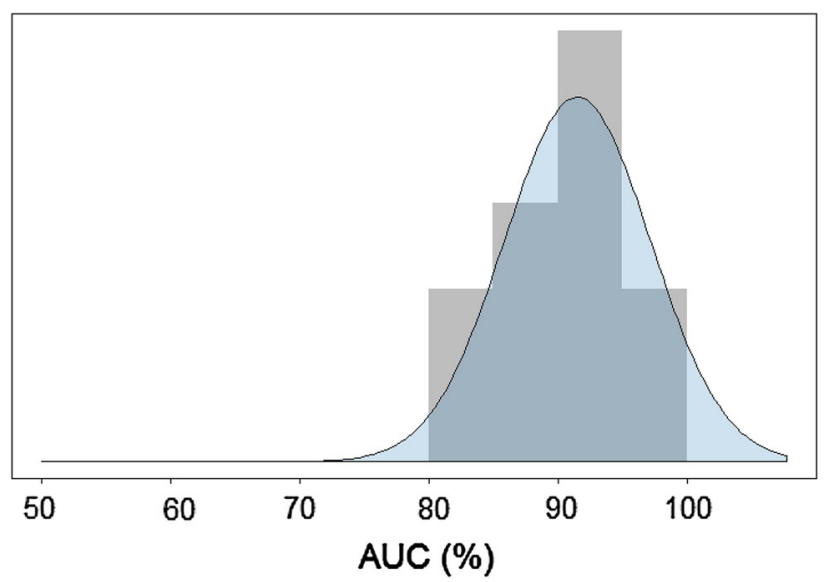

Fig. 2 Distribution of the 12 AUC scores measured on the outer validation set of the Monte Carlo cross-validation (MCCV) around the mean score of $91 \%$ 
strong stability in feature selection and a very good level of classification performance.

\section{Discussion}

This is a proof of concept study illustrating the ability of a specific ML approach, the XGBoost algorithm, to classify subjects in two distinct classes or categories, healthy/typical versus patients with epilepsy/atypical, according to their language representation, as determined with fMRI. This statistical method does not require manipulation of data and uses neurophysiological features reflecting the amount of activated language regions, for two main processes: semantic and phonological. Our results showed that a specific subset best distinguished the two categories of participants, namely the subset SEM_LH BA_47-21, showing that the left fronto-temporal activation induced by the SEM task was the most relevant to classify patients. This result can be discussed in the framework of current debates on language representation and reorganization in focal epilepsy [3]. Our result reflects reorganization of language networks in the predominant left hemisphere for language [4], and this can be considered as a specific 'atypical' profile of language representation. Indeed, the majority of individuals, mainly healthy, show 'typical' language representation with the left hemisphere predominant for language [4]. The majority of patients with focal epilepsy show higher variability of language representation within and between hemispheres, known as 'atypical' profiles, induced by the chronic development of the epileptic activity. Although many atypical profiles were described [42], three of them are more frequently observed (see [3]): (a) atypical inter-hemispheric representation with complete displacement of language areas from the left to the right regions [14, 53, 54]; (b) atypical inter-hemispheric representation with only partial displacement of language regions to the right hemisphere $[17,55]$; and (c) atypical intra-hemispheric reorganization of language networks within the predominant, left hemisphere for language. This latter profile of reorganization fits well with our result, indicating that the best distinction between patients and healthy is based on changes occurring in the predominant left hemisphere for language. Indeed, an intrahemispheric reorganization of language networks with supplementary or additional recruitment of fronto (BA47)temporal (BA21) regions might occur in patients to maintain a correct level of language performance. The neurophysiological biomarker that seems to distinguish patients from healthy individuals at an intra-hemispheric level, is the activation of two crucial integrative regions-one frontal, the BA 47 and the other temporal, the BA 21-both responsible for semantic processing [56]. Their effect was located in the same left hemisphere, hence providing an intra-hemispheric biomarker of the distinction patients versus controls. Importantly, these regions should be considered in interaction rather than separately, given that they belong to neurocognitive models of language mainly for semantic processing, involved in retrieval, access, selection, online maintenance and activation of lexico-semantic representations [57]. Specifically, the BA 47 in the left inferior frontal gyrus is related to retrieval and selection of semantic features and supports controlled access to stored semantic representations [58]. The posterior middle temporal gyrus, BA 21, is generally implicated in the representation of verbal semantic information [59]. These two regions are anatomically and functionally connected. A reciprocal modulatory effect from the left inferior frontal gyrus (BA 47) to the left posterior middle temporal gyrus (BA 21) was shown by using dynamic causal modeling (DCM), suggesting top-down influences of the frontal cortex on the retrieval of semantic representations. In the opposite direction, the effective connectivity analyses also showed modulatory effects from the left BA 21 to the left BA 47, suggesting that posterior temporal regions provide relevant associations in verbal semantic memory to IFG for the purpose of retrieval [60]. In terms of anatomical connectivity, these regions are connected by white matter fibers such as the left inferior fronto-occipital fasciculus, left anterior thalamic radiation and left uncinate, and considered as the anatomical skeleton of the semantic network [61]. Overall, all these functional and anatomical data suggest that left fronto-temporal regions revealed by activation of BA 47 and BA 21, both part of the semantic network, are reorganized in patients with epilepsy compared to healthy subjects. Given that the majority of these patients show dysfunctions of temporal regions, this could explain why the semantic system is particularly sensitive, disrupted and reorganized in patients with epilepsy. An important contribution to this reorganization is added by the interaction between semantic language and memory processes, given that a part of the anatomical subjacent regions are common to both language and memory. This also explains why these two cognitive functions are increasingly examined together rather than separately [62]. These observations are reflected and confirmed by the neuropsychological testing, showing that these patients frequently have semantic (both language and memory) deficits. In conclusion, this biomarker of intrahemispheric reorganization of fronto-temporal semantic networks revealed by the XGBoost algorithm for distinguishing patients from controls is in agreement with our knowledge on semantic processing. This result is in agreement with data from patients with epilepsy. It holds for the results obtained from invasive electrical stimulation [63] or noninvasive fMRI mapping [15, 64]. Specifically, patients with epilepsy show modification of language networks and they demonstrate a higher recruitment of the left hemispheric areas (inside and/or outside the 'eloquent networks') to 
ensure efficient language processing [64]. Nevertheless, compared to more visible inter-hemispheric profiles, the intra-hemispheric reorganization of language activity is more difficult to observe with classical fMRI statistical analyses. Mbwana et al. [64] suggested, for instance, that fMRI comparisons between patients and healthy subjects are constrained by a priori assumptions and reliance on preselection of cerebral regions and that the incidence of intrahemisphere reorganization may be underestimated or masked. Based on these assumptions, we suggest that the ML-XGBoost algorithm could be a useful tool to detect the intra-hemispheric atypical reorganization patterns, more difficult to assess in patients, but having a major role in the neuroplasticity of language in patients with epilepsy. In terms of suitable tasks to map language networks, our results show that compared to phonological task, the semantic task is more reliable for classifying patients, even if both tasks activate fronto-temporal regions. The advantage of using a semantic task is that this task induces a more spread-out activation within frontal and temporal language networks. This assumption is in agreement with the findings by Billingsley et al. [9], showing that language reorganization in patients is mainly revealed by a semantic task, whereas a phonological task results in more specific prefrontal activation. Indeed the predictive capacity of postsurgical language outcome depends significantly on the regional location of brain activity. Moreover, a specific question raised by many investigations on language representation and lateralization is whether it is necessary to map the entire language system including frontal and temporal regions for this answer or whether only a partial mapping of frontal or temporal regions is sufficient to reveal the predominant hemisphere for language. Our winning subset SEM_LH BA_47-21 suggests that the robust classification of patients requires information on a larger fronto-temporal network which is efficiently revealed by a SEM task. In terms of clinical impact, we claim that the differential intra-hemispheric reorganization as reflected by SEM_LH BA_47-21 could suggest that left fronto-temporal regions are 'sensitive-to-surgery' and should be spared during surgery to avoid postsurgical language deficits. Methodologically, we claim that the XGBoost algorithm used in this study is able to compare cognitively plausible patterns (feature subsets) and highlight the best one, and able to separate categories of participants.

\section{Conclusions}

The ML-XGBoost is a powerful statistical method of classification which detects nonlinear patterns in datasets with missing values. It shows significant potential for classifying patients with epilepsy based on the cerebral region, hemisphere and processing of their language representation. One subset, or a specific combination of features, the SEM_LH BA_47-21, was the most powerful, for identifying patients. The importance of this particular subset is plausible given the cognitive and clinical observations made with these patients.

Acknowledgements This work was partially funded for Grenoble MRI facility IRMaGe, by the French program 'Investissement d'Avenir' run by the 'Agence Nationale pour la Recherche': Grant 'Infrastructure d'Avenir en Biologie Santé' (ANR-11-INBS-0006).

\section{Compliance with ethical standards}

Conflict of interest The authors declare no conflicts of interest.

Open Access This article is distributed under the terms of the Creative Commons Attribution 4.0 International License (http://crea tivecommons.org/licenses/by/4.0/), which permits unrestricted use, distribution, and reproduction in any medium, provided you give appropriate credit to the original author(s) and the source, provide a link to the Creative Commons license, and indicate if changes were made.

\section{References}

1. Wieser H, Engel J, Pea Williamson (1993) Surgically remediable temporal lobe syndromes. Surgical treatment of the epilepsies. Raven Press, New York, pp 49-63

2. Springer J, Binder J, Hammeke T, Swanson S, Frost J, Bellgowan P, Brewer C, Perry H, Morris G, Muller W (1999) Language dominance in neurologically normal and epilepsy subjects. A functional MRI study Brain. A J Neurol 122(11):20033-22045

3. Baciu M, Perrone-Bertolotti M (2015) What do patients with epilepsy tell us about language dynamics? A review of fMRI studies. Rev Neurosci 26(3):323-341

4. Josse G, Tzourio-Mazoyer N (2004) Hemispheric specialization for language. Brain Res Rev 44(1):1-12

5. Noachtar S, Borggraefe I (2009) Epilepsy surgery: a critical review. Epilepsy Behav 15(1):66-72

6. Wrench JM, Matsumoto R, Inoue Y, Wilson SJ (2011) Current challenges in the practice of epilepsy surgery. Epilepsy Behav (in Press, Corrected Proof)

7. Wada JA, Clarke R, Hamm A (1975) Cerebral hemispheric asymmetry in humans: cortical speech zones in 100 adult and 100 infant brains. Arch Neurol 32(4):239-246

8. Sharan A, Ooi YC, Langfitt J, Sperling MR (2011) Intracarotid amobarbital procedure for epilepsy surgery. Epilepsy Behav 20(2):209-213

9. Billingsley RL, McAndrews MP, Crawley AP, Mikulis DJ (2001) Functional MRI of phonological and semantic processing in temporal lobe epilepsy. Brain 124(6):1218

10. Cousin E, Baciu M, Pichat C, Kahane P, Le Bas JF (2008) Functional MRI evidence for language plasticity in adult epileptic patients: preliminary results. Neuropsychiatr Dis Treat 4(1):235

11. Berl MM, Balsamo LM, Xu B, Moore EN, Weinstein SL, Conry JA, Pearl PL, Sachs BC, Grandin CB, Frattali C (2005) Seizure focus affects regional language networks assessed by fMRI. Neurology 65(10): 1604

12. Thiel A, Habedank B, Herholz K, Kessler J, Winhuisen L, Haupt WF, Heiss W-D (2006) From the left to the right: how the brain compensates progressive loss of language function. Brain Lang 98(1):57-65 
13. Rosenberger LR, Zeck J, Berl MM, Moore EN, Ritzl EK, Shamim S, Weinstein SL, Conry JA, Pearl PL, Sato S (2009) Interhemispheric and intrahemispheric language reorganization in complex partial epilepsy. Neurology 72:1830

14. Dijkstra KK, Ferrier CH (2013) Patterns and predictors of atypical language representation in epilepsy. J Neurol Neurosur Psychiatry. doi:10.1136/jnnp-2012-303141

15. Perrone-Bertolotti M, Zoubrinetzky R, Gt Yvert, Le Bas JF, Baciu M (2012) Functional MRI and neuropsychological evidence for language plasticity before and after surgery in one patient with left temporal lobe epilepsy. Epilepsy Behav 23(1):81-86

16. Baciu M, Watson J, Maccotta L, McDermott K, Buckner R, Gilliam F, Ojemann J (2005) Evaluating functional MRI procedures for assessing hemispheric language dominance in neurosurgical patients. Neuroradiol 47(11):835-844

17. Baciu M, Watson J, McDermott K, Wetzel R, Attarian H, Moran C, Ojemann J (2003) Functional MRI reveals an interhemispheric dissociation of frontal and temporal language regions in a patient with focal epilepsy. Epilepsy Behav 4(6):776-780

18. Gazit T, Andelman F, Glikmann-Johnston Y, Gonen T, Solski A, Shapira-Lichter I, Ovadia M, Kipervasser S, Neufeld MY, Fried I, Hendler T, Perry D (2016) Probabilistic machine learning for the evaluation of presurgical language dominance. J Neurosurg 125(2):481-493

19. Abbott DF, Waites AB, Lillywhite LM, Jackson GD (2010) fMRI assessment of language lateralization: an objective approach. Neuroimage 50(4):1446-1455

20. Spritzer SD, Hoerth MT, Zimmerman RS, Shmookler A, Hoffman-Snyder CR, Wellik KE, Bart D, Wingerchuk DM (2012) Determination of hemispheric language dominance in the surgical epilepsy patient: diagnostic properties of functional magnetic resonance imaging. Neurologist 18(5):329-331

21. Alvarez A, Sierra B, Arruti A, Lopez-Gil J-M, Garay-Vitoria N (2015) Classifier subset selection for the stacked generalization method applied to emotion recognition in speech. Sensors 16(1):21

22. Kaufmann T, Elvsåshagen T, Alnæs D, Zak N, Pedersen PØ, Norbom LB, Quraishi SH, Tagliazucchi E, Laufs H, Bjørnerud A, Malt UF, Andreassen OA, Roussos E, Duff EP, Smith SM, Groote IR, Westlye LT (2016) The brain functional connectome is robustly altered by lack of sleep. NeuroImage 127:324-332

23. Besga A, Ortiz L, Fernandez A, Maestu F, Arrazola J, Gil-Gregorio P, Fuentes M, Ortiz T (2015) Structural and functional patterns in healthy aging, mild cognitive impairment, and Alzheimer disease. Alzheimer Dis Assoc Disord 24(1):1-10

24. Steele VR, Rao V, Calhoun VD, Kiehl KA (2017) Machine learning of structural magnetic resonance imaging predicts psychopathic traits in adolescent offenders. NeuroImage 145(B):265-269

25. Munsell BC, Wee C-Y, Keller SS, Weber B, Elger C, da Silva LAT, Nesland T, Styner M, Shen D, Bonilha L (2015) Evaluation of machine learning algorithms for treatment outcome prediction in patients with epilepsy based on structural connectome data. NeuroImage 118(1):219-230

26. Chen T, Guestrin C (2016) Xgboost: a scalable tree boosting system. In: Proceedings of the 22Nd ACM SIGKDD international conference on knowledge discovery and data mining. ACM, pp 785-794

27. Friedman JH (2001) Greedy function approximation: a gradient boosting machine. Ann Stat 29(5):1189-1232

28. Chen T, He T (2014) Higgs boson discovery with boosted trees. In: HEPML@ NIPS, pp 69-80

29. Möller A, Ruhlmann-Kleider V, Leloup C, Neveu J, PalanqueDelabrouille N, Rich J, Carlberg R, Lidman C, Pritchet C (2016) Photometric classification of type Ia supernovae in the
SuperNova Legacy Survey with supervised learning. J Cosmol Astropart Phys 12:008

30. Tamayo D, Silburt A, Valencia D, Menou K, Ali-Dib M, Petrovich C, Huang CX, Rein H, van Laerhoven C, Paradise A (2016) A machine learns to predict the stability of tightly packed planetary systems. Astrophys J Lett 832(2):L22

31. Cousin E, Peyrin C, Pichat Cd, Lamalle L, Le Bas J-F, Baciu M (2007) Functional MRI approach for assessing hemispheric predominance of regions activated by a phonological and a semantic task. Eur J Radiol 63(2):274-285

32. Baciu M, Kahane P, Minotti L, Charnallet A, David D, Le Bas JF, Segebarth C (2001) Functional MRI assessment of the hemispheric predominance for language in epileptic patients using a simple rhyme detection task. Epileptic Disord 3:117-124

33. Bahn MM, Lin W, Silbergeld DL, Miller JW, Kuppusamy K, Cook RJ, Hammer G, Wetzel R, Cross D 3rd (1997) Localization of language cortices by functional MR imaging compared with intracarotid amobarbital hemispheric sedation. AJR Am J Roentgenol 169(2):575-579

34. Friston KJ, Zarahn E, Josephs O, Henson RNA, Dale AM (1999) Stochastic designs in event-related fMRI. NeuroImage 10(5):607-619

35. Friston KJ, Holmes AP, Worsley KJ, Poline JP, Frith CD, Frackowiak RSJ (1995) Statistical parametric maps in functional imaging: a general linear approach. Hum Brain Mapp 2(4): $189-210$

36. Natekin A, Knoll A (2013) Gradient boosting machines, a tutorial. Front neurorobotics 7:21

37. He X, Pan J, Jin O, Xu T, Liu B, Xu T, Shi Y, Atallah A, Herbrich R, Bowers S (2014) Practical lessons from predicting clicks on ads at facebook. In: Proceedings of the eighth international workshop on data mining for online advertising. ACM, pp 1-9

38. Caruana R, Niculescu-Mizil A (2006) An empirical comparison of supervised learning algorithms. In: Proceedings of the 23rd international conference on Machine learning. ACM, pp 161-168

39. Li J, Cheng K, Wang S, Morstatter F, Trevino RP, Tang J, Liu H (2016) Feature selection: a data perspective. arXiv:160107996

40. Raschka S (2015) Python machine learning. Packt Publishing Ltd, Birmingham

41. Goldmann RE, Golby AJ (2005) Atypical language representation in epilepsy: implications for injury-induced reorganization of brain function. Epilepsy Behav 6(4):473-487

42. Berl MM, Zimmaro LA, Khan OI, Dustin I, Ritzl E, Duke ES, Sepeta LN, Sato S, Theodore WH, Gaillard WD (2014) Characterization of atypical language activation patterns in focal epilepsy. Ann Neurol 75(1):33-34

43. Pedregosa F, Varoquaux G, Gramfort A, Michel V, Thirion B, Grisel O, Blondel M, Prettenhofer P, Weiss R, Dubourg V (2011) Scikit-learn: machine learning in Python. J Mach Learn Res 12:2825-2830

44. Cawley GC, Talbot NL (2010) On over-fitting in model selection and subsequent selection bias in performance evaluation. J Mach Learn Res 11:2079-2107

45. Friedman JH (2002) Stochastic gradient boosting. Comput Stat Data Anal 38(4):367-378

46. Dubitzky W, Granzow M, Berrar DP (2007) Fundamentals of data mining in genomics and proteomics. Springer, Berlin

47. Xu QS, Liang YZ (2001) Monte Carlo cross validation. Chemometr Intell Lab Syst 56(1):1-11

48. Nowotny T (2014) Two challenges of correct validation in pattern recognition. Front Robot AI 1:5

49. Friedman J, Hastie T, Tibshirani R (2001) The elements of statistical learning. Springer series in statistics, vol 1. Springer, Berlin 
50. Provost F, Fawcett T, Kohavi R (1998) The case against accuracy estimation for comparing induction algorithms. In: Proceedings of the fifteenth international conference on machine learning. Morgan Kaufmann, pp 445-453

51. Ling CX, Huang J, Zhang H (2003) AUC: a better measure than accuracy in comparing learning algorithms. In: Xiang Y, Chaibdraa B (eds) Conference of the Canadian society for computational studies of intelligence. Springer, Berlin, pp 329-341

52. Hernández-Orallo J, Flach P, Ferri C (2012) A unified view of performance metrics: translating threshold choice into expected classification loss. J Mach Learn Res 13:2813-2869

53. Gaillard WD, Balsamo L, Xu B, Grandin C, Braniecki S, Papero P, Weinstein S, Conry J, Pearl P, Sachs B (2002) Language dominance in partial epilepsy patients identified with an fMRI reading task. Neurology 59(2):256-265

54. Thivard L, Hombrouck J, du Montcel ST, Delmaire C, Cohen L, Samson S, Dupont S, Chiras J, Baulac M, Lehéricy S (2005) Productive and perceptive language reorganization in temporal lobe epilepsy. Neuroimage 24(3):841-851

55. Ries M, Boop FA, Griebel ML, Zou P, Phillips NS, Johnson SC, Williams J, Helton KJ, Ogg RJ (2004) Functional MRI and Wada determination of language lateralization: a case of crossed dominance. Epilepsia 45(1):85-89

56. Binder JR, Desai RH, Graves WW, Conant LL (2009) Where is the semantic system? A critical review and meta-analysis of 120 functional neuroimaging studies. Cereb Cortex 19(12): 2767-2796

57. Ardila A, Bernal B, Rosselli M (2016) How localized are language brain areas? A review of Brodmann areas involvement in oral language. Arch Clin Neuropsychol 31(1):112-122

58. Badre D, Wagner AD (2004) Selection, integration, and conflict monitoring: assessing the nature and generality of prefrontal cognitive control mechanisms. Neuron 41(3):473-487

59. Booth JR, Burman DD, Meyer JR, Gitelman DR, Parrish TB, Mesulam M (2002) Modality independence of word comprehension. Hum Brain Mapp 16(4):251-261

60. Fan LY, Lee SH, Chou TL (2010) Interaction between brain regions during semantic processing in Chinese adults. Lang Linguist 11(1):159-182

61. Han Z, Ma Y, Gong G, He Y, Caramazza A, Bi Y (2013) White matter structural connectivity underlying semantic processing: evidence from brain damaged patients. Brain 136(10):2952-2965

62. Bertolotti M, Girard C, Cousin E, Vidal JR, Pichat C, Kahane P, Baciu M (2015) NEREC, an effective brain mapping protocol for combined language and long-term memory functions. Epilepsy Behav 53:140-148
63. Ojemann G, Ojemann J, Lettich E, Berger M (1989) Cortical language localization in left, dominant hemisphere. An electrical stimulation mapping investigation in 117 patients. J Neurosurg 71(3):316

64. Mbwana J, Berl MM, Ritzl EK, Rosenberger L, Mayo J, Weinstein S, Conry JA, Pearl PL, Shamim S, Moore EN (2009) Limitations to plasticity of language network reorganization in localization related epilepsy. Brain 132(2):347

L. Torlay is a computer science engineer, specialist in predictive statistical analyses, machine learning, and their implementation into applications and technological tools. Specifically, he develops explanatory or predictive models of cognitive functions in healthy subject and patients presenting cerebral lesions.

Dr. M. Perrone-Bertolotti is lecturer in Cognitive Psychology. She investigates the cognitive mechanism and the neural correlates of language processing in interaction with others cognitive functions (such as executive control, attention, action and verbal thought). Her work aims to understand how we can improve language comprehension and production by training other cognitive functions (reshaping the language network). To do so, she uses a multidisciplinary and a multimethodological approach.

Dr. E. Thomas is lecturer in biomedical sciences, currently carrying out computational and experimental work on the following projects: (a) muscular synergies during motor control that permit a reduction in the number of degrees of freedom involved when executing a movement; (b) machine learning methods for identifying ensemble changes in the nervous system, and (c) modifications of movement patterns with aging.

Dr. M. Baciu is a professor of cognitive neuroscience, interested in developing novel neurocognitive, integrative and dynamic models of neuroplasticity for language and memory, in physiological and pathological condition (mainly for focal epilepsy and post-stroke aphasia) as well as during normal and pathological aging. Dr. Baciu's training and experience as both a neuroscientist and neurologist enable her to do both basic and clinical research, for which she draws on the fields of experimental psychology, neuropsychology and neuroimaging. 